\title{
Simultaneous determination of multiple marker constituents in concentrated Gegen Tang granule by high performance liquid chromatography
}

\author{
Jingzheng Song, Quanbin Han, Chunfeng Qiao, Yuekeung Yip and \\ Hongxi $\mathrm{Xu}^{*}$
}

Address: Chinese Medicine Laboratory, Hong Kong Jockey Club Institute of Chinese Medicine, Shatin, NT, Hong Kong SAR, China

Email: Jingzheng Song - jzsong@hkjcicm.org; Quanbin Han - qbhan@hkjcicm.org; Chunfeng Qiao - cfqiao@hkjcicm.org;

Yuekeung Yip - masonyip@hkjcicm.org; Hongxi Xu* - xuhongxi@hkjcicm.org

* Corresponding author

Published: 20 June 2007

Chinese Medicine 2007, 2:7 doi:10.1186/1749-8546-2-7

This article is available from: http://www.cmjournal.org/content/2/l/7

C) 2007 Song et al; licensee BioMed Central Ltd.

This is an Open Access article distributed under the terms of the Creative Commons Attribution License (http://creativecommons.org/licenses/by/2.0), which permits unrestricted use, distribution, and reproduction in any medium, provided the original work is properly cited.
Received: I September 2006

Accepted: 20 June 2007

\begin{abstract}
Background: Concentrated Gegen Tang (GT) granule is a widely available traditional Chinese medicinal product for the treatment of cold and flu. There was no reliable analytical method available for the quality assessment of GT granules.

Methods: An HPLC method with an Agilent Zorbax SB-Phenyl Stablebond column $(250 \times 4.6 \mathrm{~mm}$, $5 \mu \mathrm{m})$ was developed and validated. The mobile phase gradient was a mixture of $0.1 \%$ trifluoroacetic acid (TFA) in acetonitrile (ACN) and $0.1 \%$ TFA in water. The detection with a diodearray detector was set at 207, 230, 250 and $275 \mathrm{~nm}$. Seven components, namely puerarin, daidzein, paeoniflorin, cinnamic acid, glycyrrhizin, ephedrine and pseudoephedrine were selected as marker compounds for the evaluation.

Results: The regression equations revealed good linear relationships (correlation coefficients: $0.9994-0.9998$ ) between the peak areas and concentrations. The recovery was between $98.8 \%$ and $101.7 \%$ with good precision and accuracy. The quality of GT granule from four different manufacturers was evaluated with this newly developed method. Samples from four manufacturers showed similar profiles but contents of the detected markers varied significantly among manufacturers and batches.

Conclusion: A new method using high performance liquid chromatography (HPLC) has been developed for simple and reliable quality control of commercial concentrated GT granules. Sensitivity was increased by multi-wavelength detection. The contents of selected marker components in GT granules varied significantly among manufacturers and batches, making it necessary to evaluate the quality of concentrated GT granules in the market.
\end{abstract}

\section{Background}

Listed in Shanghan Lun (Discussion of Cold-induced Disorders) by Zhang Zhonging of the Han Dynasty, Gegen Tang
(GT), a decoction of Radix Pueraria Lobatae (Gegen), is a classical formula of traditional Chinese medicine. It is widely used in China and South East Asia for treating cold 
and flu [1]. Modern pharmacological studies have revealed that it can alleviate upper respiratory tract diseases, trigeminal neuralgia, lumbar muscle strains, tension headaches, arthralgia, ischemic stroke, rheumatoid arthritis, dysentery and nettle rash. It also has antiviral, anti-pathogen, anti-inflammatory, and anticoagulant activities [2-9]. Some studies indicate that these effects are attributed to the major components in this preparation, such as puerarin, daidzein, paeoniflorin, cinnamic acid, glycyrrhizin, ephedrine and pseudoephedrine [2,10-12], the chemical structures of which are shown in Figure 1. In recent years, the formula of GT has been developed into a concentrated granule form which has advantage over the traditional decoction.

The formula of GT consists of seven herbs (Table 1). According to traditional Chinese Medicine theories, Radix Puerariae (Gegen) is the 'primary' herb in the formula, whereas Herba Ephedrae (Mahuang) and Ramulus Cinnamomi Cassiae (Guizhi) are 'deputies' to help release the 'primary' herb and relieve the muscle layer. Radix Paeoniae Alba (Baishao), Rhizoma Zingiberis Recens (Shengjiang) and Fructus Zizyphi Jujubae (Dazao) are 'assistant' herbs while Radix Glycyrrhizae (Gancao) is the 'envoy' [1].

The quality of the raw herbs is one of the major factors for the quality of proprietary preparations of traditional Chinese medicines. For example, roots from Puerariae lobata (Willd.) Ohwi and P. thomsonii Benth are used as the

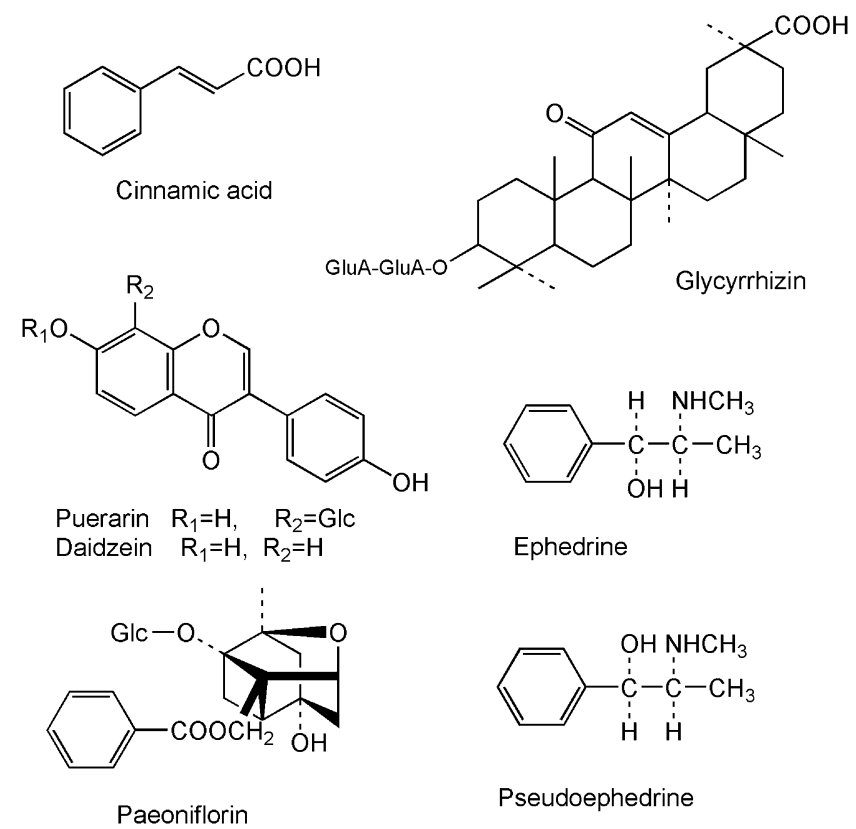

Figure I

Chemical structures of seven chemical markers in Gegen Tang granule. source of Radix Puerariae. However, the contents of puerarin and daidzein, two major marker components in Radix Puerariae Lobatae, were 8-fold and 5-fold higher than those in Radix Puerariae Thomsonii (Fenge) respectively [13]. An interesting study revealed that Radix Puerariae Lobatae was much more effective in relieving fever than Radix Puerariae Thomsonii [13]. Furthermore, the regions where the raw materials were collected and the season when they were collected may also affect the quality of the preparations. As few analytical methods are available for the quality control of GT granules, the quality of concentrated GT granules in the market still remain questionable.

As a popular tool for the quality control of traditional Chinese medicines, high performance liquid chromatography (HPLC) was used for the determination of GT. However, its procedure was very complicated $[14,15]$. A micellar electrokinetic chromatography (MEKC) method was also used for the determination of seven marker compounds in GT granules [16]. Between the two methods, the precision and accuracy of the MEKC method were not satisfactory for quantitative analysis and its sensitivity was inferior to that of HPLC.

As concentrated GT granule is popularly used as a nonprescriptive drug in China and East Asia, its quality control is vitally important. In this paper, we report an HPLC method first developed and validated for the quality control of GT granule. While often used as specific marker compounds for conventional quality control of the respective herbs as well as proprietary Chinese medicinal preparations, seven components were simultaneously determined in this new method. Extraction efficiency of these markers was optimized through comparisons of extraction of granules with different solvents and time intervals. Finally, the quality of GT granules was evaluated under the optimized HPLC method.

\section{Methods}

\section{Material and reagents}

Fourteen batches of concentrated GT granules manufactured by four different manufacturers (code named A, B, $\mathrm{C}$ and $\mathrm{D}$ ) were purchased from local pharmacies in Hong Kong. The reference standards of puerarin, daidzein, paeoniflorin, cinnamic acid and glycyrrhizin, were purchased from the National Institute for the Control of Pharmaceutical and Biological Products of China (Beijing, China). Ephedrine and pseudoephedrine were purchased from Sigma Chemical Co. (St. Louis, USA). The purity for all reference standards was over $98 \%$. Trifluroacetic acid (HPLC grade) was purchased from International Laboratory (San Bruno, USA). HPLC-grade methanol and acetonitrile were obtained from Duksan chemical Co. (Kyungki-Do, Korea). HPLC-grade water used throughout 
Table I: Herbal composition and daily dosage of Gegen Tang granule from four manufacturers

\begin{tabular}{|c|c|c|c|c|}
\hline Ingredients & A* & B & C & D \\
\hline Radix Puerarae (Gegen) & - & $22.7 \%$ & $32.0 \%$ & $21.4 \%$ \\
\hline Herba Ephedrae (Mahuang) & - & $16.6 \%$ & $16.0 \%$ & $16.1 \%$ \\
\hline Radix Glycyrrhizae (Gancao) & - & $10.7 \%$ & $8.0 \%$ & $10.7 \%$ \\
\hline Radix Paeoniae Alba (Baishao) & - & $11.0 \%$ & $12.0 \%$ & $10.7 \%$ \\
\hline Ramulus Cinnamomi Cassiae (Guizhi) & - & $11.0 \%$ & $12.0 \%$ & $10.7 \%$ \\
\hline Rhizoma Zingiberis Recens (Shengjiang) & - & $16.6 \%$ & $4.0 \%$ & $16.1 \%$ \\
\hline Frutus Jujubae (Dazao) & - & $11.0 \%$ & $16.0 \%$ & $14.3 \%$ \\
\hline Daily dosage & $2 \times 4 g$ & $2 \times 5 g$ & $3 \times 3 g$ & $2 \times 5 g$ \\
\hline
\end{tabular}

The percentage of each herbal ingredient was collected from the insert (B and C), or calculated after normalized total to $100 \%$ (D).

*The composition of $A$ is unknown.

the study was obtained from a Milli-Q Reagent Water System (Millopore, USA).

\section{Instruments and chromatographic conditions}

All separations were performed on a Waters 2695 Separations Module HPLC system equipped with a quaternary pump, autosampler, column temperature controller, an online degasser and a Waters 2996 Photodiode Array Detector (PDA). The analytical column was an Agilent Zorbax SB-Phenyl Stablebond column $(250 \times 4.6 \mathrm{~mm}, 5$ $\mu \mathrm{m})$.

The mobile phase was composed of (A) water, (B) $0.1 \%$ TFA in water and (C) $0.1 \%$ TFA in ACN (A-B-C: 0 min 75:20:5; $25 \min 60: 20: 20 ; 50 \min 17: 20: 63 ; \mathrm{v} / \mathrm{v} / \mathrm{v})$. The flow rate was $1.0 \mathrm{~mL} / \mathrm{min}$. The column effluents were simultaneously monitored at $207 \mathrm{~nm}$ (for ephedrine and pseudoephedrine), $230 \mathrm{~nm}$ (for paeoniflorin), $275 \mathrm{~nm}$ (for cinnamic acid) and $250 \mathrm{~nm}$ (for other components). Content of each component was calculated on the basis of the absorbance at each respective selected wavelength. Injection volume was $10 \mu \mathrm{L}$. The column temperature was controlled at $25^{\circ} \mathrm{C}$.

\section{Preparation of standard solutions}

The standard stock solutions $(1 \mathrm{mg} / \mathrm{mL})$ were prepared by dissolving $5 \mathrm{mg}$ of each reference standard to $5 \mathrm{~mL}$ of methanol. The stock solutions were stored at $4{ }^{\circ} \mathrm{C}$ and remained stable for at least one month. The working standard solutions containing each of the seven reference standards were prepared by diluting the standard stock solutions to give serial concentrations within ranges listed in Table 2. The standard curve was analyzed with Excel using the linear least-squares regression equation derived from the peak areas.

Recovery of the seven marker components from GT granule was determined by spiking known amount of reference standards at three different concentrations (Table 3) to $20 \mathrm{mg}$ of GT granule with known contents of the seven markers.

\section{Sample preparation}

Ten grams of each GT granule sample was ground to fine powder. Forty milligrams of the fine powder was accurately weighed and dissolved in $10 \mathrm{~mL}$ of $60 \%$ methanol $(\mathrm{v} / \mathrm{v})$ by ultrasonication for $30 \mathrm{~min}$. After being cooled down to room temperature, the sample solution was centrifuged at $13000 \mathrm{rpm}$ for $5 \mathrm{~min}$ and the supernatant was filtered through a $0.2 \mu \mathrm{m}$ syringe membrane filter. Ten microliters of the filtrate was injected into the HPLC system for analysis.

Table 2: Regression equation, correlation coefficients, linearity ranges and limit of detection and quantitation for the markers of Gegen Tang granule

\begin{tabular}{|c|c|c|c|c|c|c|c|}
\hline Compounds & $\begin{array}{c}\text { Regression } \\
\text { equation }\end{array}$ & $\begin{array}{c}\text { Linear range } \\
(\mu \mathrm{g} / \mathrm{mL})\end{array}$ & $\mathbf{R}^{2}$ & LOD (ng/mL) & LOQ (ng/mL) & $\begin{array}{c}\text { Intra-day RSD\% } \\
(n=6)\end{array}$ & $\begin{array}{c}\text { Inter-day RSD\% } \\
(n=3)\end{array}$ \\
\hline Puerarin & $y=40149 x-5483$ & $1.70-69.70$ & 0.9997 & 6.21 & 20.70 & 0.83 & 0.78 \\
\hline Paeoniflorin & $y=13018 x+3168$ & $1.07-64.36$ & 0.9996 & 19.64 & 65.46 & 0.99 & 0.45 \\
\hline Cinnamic acid & $y=68413 x+2472$ & $0.15-5.81$ & 0.9998 & 3.72 & 12.40 & 0.61 & 0.60 \\
\hline Daidzain & $y=44698 x+350$ & $0.06-4.96$ & 0.9997 & 5.58 & 18.60 & 0.33 & 1.83 \\
\hline Glycyrrhizin & $y=5695 x-3089$ & $1.07-85.26$ & 0.9998 & 45.47 & 151.56 & 0.10 & 1.70 \\
\hline Ephedrine & $y=21411 x+1949$ & $1.08-86.13$ & 0.9998 & 3.82 & 12.73 & 0.52 & 1.55 \\
\hline Pseudoephedrine & $y=30269 x-41321$ & $2.98-119.13$ & 0.9996 & 2.71 & 9.03 & 0.37 & 0.41 \\
\hline
\end{tabular}

All regression results are statistically significant $(P<0.05)$ 
Table 3: Recovery of seven markers in Gegen Tang sample*

\begin{tabular}{|c|c|c|c|c|c|}
\hline Compounds & Contents $(\mu \mathrm{g} / \mathrm{g})$ & RSD\% $(n=6)$ & Added $(\mu \mathrm{g} / \mathrm{mL})$ & Obtained $(\mu \mathrm{g} / \mathrm{mL})$ & Recovery ( \pm RSD \%, n = 3) \\
\hline \multirow[t]{3}{*}{ Puerarin } & 3920 & 0.61 & 3.50 & 3.54 & $101.20 \pm 0.74$ \\
\hline & & & 8.75 & 8.68 & $99.26 \pm 0.43$ \\
\hline & & & 13.85 & 13.75 & $99.41 \pm 0.51$ \\
\hline \multirow[t]{3}{*}{ Paeoniflorin } & 3219 & 0.54 & 3.63 & 3.60 & $99.28 \pm 1.61$ \\
\hline & & & 9.07 & 8.99 & $99.14 \pm 1.07$ \\
\hline & & & 13.27 & 13.16 & $99.33 \pm 0.51$ \\
\hline \multirow[t]{3}{*}{ Cinnamic acid } & 317 & 0.73 & 0.27 & 0.27 & $99.78 \pm 1.01$ \\
\hline & & & 0.68 & 0.68 & $100.23 \pm 1.67$ \\
\hline & & & 0.87 & 0.87 & $100.01 \pm 1.06$ \\
\hline \multirow[t]{3}{*}{ Daidzain } & 283 & 1.14 & 0.19 & 0.19 & $100.35 \pm 1.10$ \\
\hline & & & 0.48 & 0.48 & $98.94 \pm 1.50$ \\
\hline & & & 0.78 & 0.77 & $98.76 \pm 0.76$ \\
\hline \multirow[t]{3}{*}{ Glycyrrhizin } & 4138 & 0.55 & 3.56 & 3.59 & $100.92 \pm 1.16$ \\
\hline & & & 8.89 & 8.93 & $100.54 \pm 0.40$ \\
\hline & & & 16.96 & 17.09 & $100.73 \pm 0.30$ \\
\hline \multirow[t]{3}{*}{ Ephedrine } & 4283 & 0.67 & 3.70 & 3.74 & $101.03 \pm 0.83$ \\
\hline & & & 9.26 & 9.30 & $100.38 \pm 1.34$ \\
\hline & & & $|4.4|$ & 14.29 & $99.22 \pm 1.35$ \\
\hline \multirow{3}{*}{ Pseudoephedrine } & 1007 & 1.21 & 0.50 & 0.96 & $101.70 \pm 1.17$ \\
\hline & & & 2.37 & 2.39 & $101.02 \pm 2.01$ \\
\hline & & & 4.19 & 4.23 & $100.71 \pm 0.72$ \\
\hline
\end{tabular}

*Sample Batch No. 4 from manufacturer D is used in this experiment.

Statistical analyses were performed using Data Analysis Toolpak Microsoft Excel for Windows. It is considered to be statistical significant when P value is less than 0.05 .

\section{Results}

Optimization of chromatographic conditions

The mobile phase was optimized through comparisons of different solvents, solvent ratio and gradient profile. Compared with other solvents, acetonitrile showed the best separation, shortest analyzing time and lowest column pressure. An acidified mobile phase could minimize peak tailing and improve resolution. A simple gradient was used for single run elution within $50 \mathrm{~min}$. A mixture of water, $0.1 \%$ TFA in water and $0.1 \%$ TFA in ACN were chosen as the mobile phase gradient. The resolution between cinnamic acid (peak 3 ) and daidzein (peak 4) was 1.3 and the resolutions between other peaks were larger than 2.0.

\section{Method Validation}

\section{Calibration}

All calibration graphs were plotted based on linear regression analysis of the integrated peak areas $(y)$ versus concentrations $(x, \mu \mathrm{g} / \mathrm{mL})$ of the seven markers in the standard solution at ten different concentrations. Results showed a good linear relationship between the peak area and concentration (Table 3 ).

The limit of detection (LOD) and limit of quantitation (LOQ) were determined with standard solution on the basis of a signal-to-noise ratio of 3 and 10 respectively.
The values of LOD and LOQ were much lower than the reported methods [13-15], indicating that the proposed method was sensitive for the determination.

Precision

The intra-day precision was evaluated by determining a standard mixture solution of the seven markers under the optimized condition six times within a day. For inter-day precision, the measurement was conducted two times per day for three consecutive days. As shown in Table 2, the intra-day and inter-day relative standard deviations (RSDs) were $0.10-0.99 \%$ and $0.41-1.83 \%$ respectively.

\section{Repeatability}

Six independently prepared sample solutions of concentrated GT granules with the same amount were analyzed and the variations within six measurements were calculated for repeatability. The measurements followed those described in sample preparation. RSDs (parameters for repeatability) ranged from 0.54 to $1.21 \%$ (Table 3), indicating that the conditions used in the quantitative analysis were satisfactory.

\section{Recovery}

Recovery studies were carried out by spiking three different concentrations of the mixed standards to the GT granules sample. The average recoveries were $99.3-101.2 \%$ for puerarin, $99.1-99.3 \%$ for paeoniflorin, $99.8-100.2 \%$ for cinnamic acid, $98.8-100.4 \%$ for daidzein, $100.5-100.9 \%$ for glycyrrhizin, $99.2-100.4 \%$ for ephedrine and $100.1-$ 
$101.7 \%$ for pseudoephedrine (Table 3). The results showed that the proposed method was accurate for the determination.

\section{Sample analysis}

The extraction efficiency was evaluated by $100 \%$ methanol, $60 \%$ methanol and water. As regards the samples from manufacturers $\mathrm{B}, \mathrm{C}$ or $\mathrm{D}$, it was shown that the extraction efficiencies were similar among these three solvents and no obvious difference was observed among them. However, in the sample from manufacturer A, the extraction efficiency achieved by $60 \%$ methanol was much higher than those by other solvents. Thus, $60 \%$ methanol was chosen for extraction in this study.

Solvent volume and sonication time were also optimized $(5,10$ and $20 \mathrm{~mL}$ of $60 \%$ methanol sonicated at 10,20 , 30 and $40 \mathrm{~min}$ ). For the samples from manufacturers B, C and D, 20 min sonication produced the best yield. For the sample from manufacturer A, 30 min sonication was needed. As regards volume of solvent, $10 \mathrm{~mL}$ produced the best yield, sensitivity and precision.
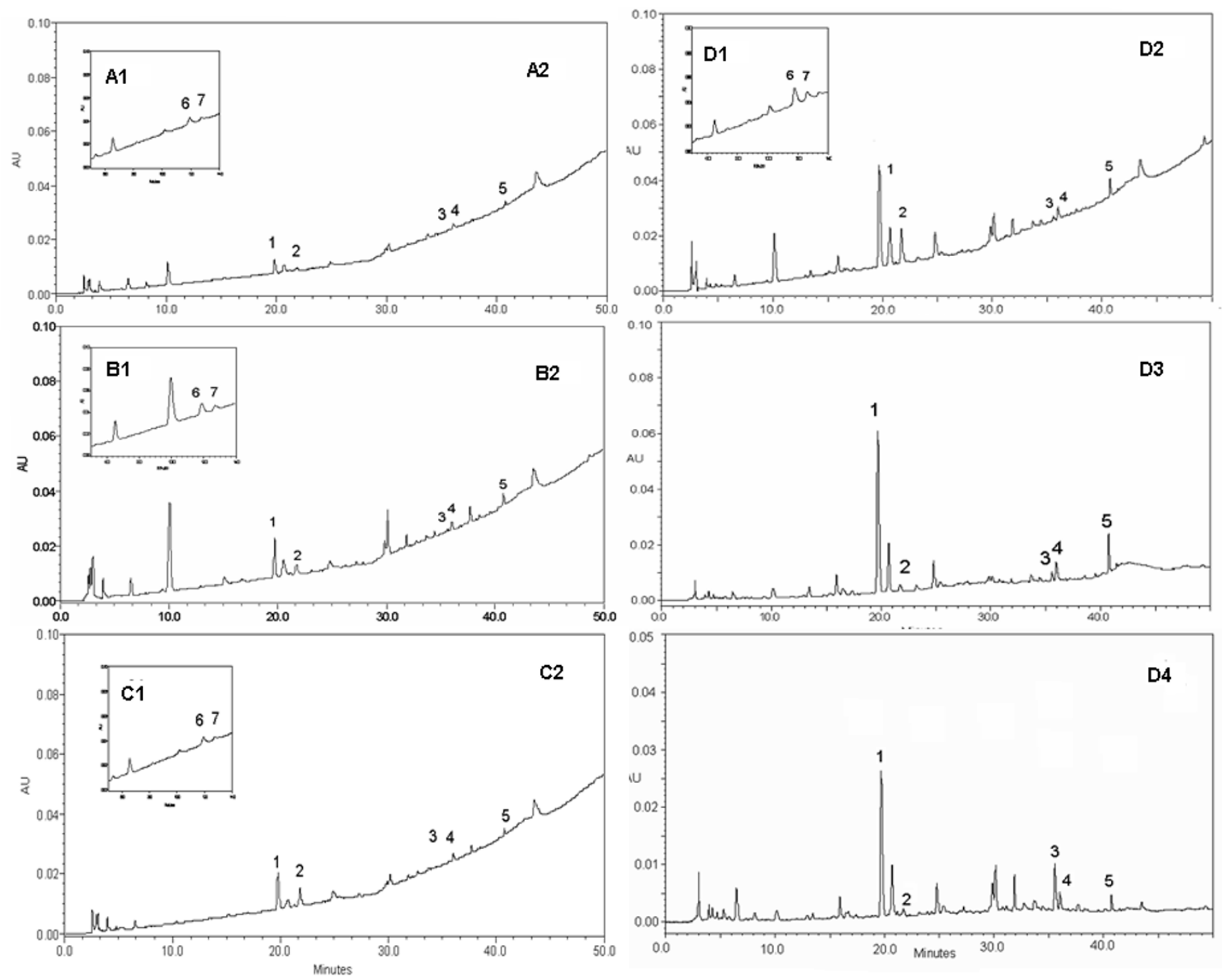

Figure 2

HPLC chromatograms of seven marker compounds detected in different samples under the developed mobile phase system at different detection wavelengths. Letters $A, B, C$ and $D$ denote the four manufacturers. Peaks: (I) Puerarin; (2) Paeoniflorin; (3) Cinnamic acid; (4) Daidzein; (5) Glycyrrhizin; (6) Ephedrine; (7) Pseudoephedrine. A2, B2, C2 and D2 are chromatograms monitored at $230 \mathrm{~nm}$ for the quantitative determination of paeoniflorin (peak 2) and also used for the comparison of different samples. AI, BI, CI and DI are chromatograms monitored at $207 \mathrm{~nm}$ for the determination of ephedrine (peak 6) and pseudoephedrine (peak 7). D3 is a chromatogram monitored at $250 \mathrm{~nm}$ for puerarin (peak I), daidzein (peak 4) and glycyrrhizin (peak 5); and D4 is a chromatogram monitored at $275 \mathrm{~nm}$ for cinnamic acid (peak 3). 
Table 4: Contents ( $\mu \mathrm{g} / \mathrm{g}$, mean $\pm \mathrm{SD}, \mathrm{n}=3$ ) of markers in Gegen Tang granule from four manufacturers

\begin{tabular}{|c|c|c|c|c|c|c|c|c|}
\hline \multicolumn{2}{|c|}{$\begin{array}{c}\text { Manufacturers and Batch } \\
\text { No. }\end{array}$} & \multirow{2}{*}{$\begin{array}{l}\text { Puerarin } \\
374.3 \pm 3.1\end{array}$} & \multirow{2}{*}{$\begin{array}{l}\text { Paeoniflorin } \\
270.5 \pm 0.6\end{array}$} & \multirow{2}{*}{$\begin{array}{l}\text { Cinnamic } \\
\text { acid }\end{array}$} & \multirow{2}{*}{$\begin{array}{l}\text { Daidzain } \\
52.1 \pm 0.4\end{array}$} & \multirow{2}{*}{$\begin{array}{l}\text { Glycyrrhizin } \\
411.4 \pm 2.8\end{array}$} & \multirow{2}{*}{$\begin{array}{l}\text { Ephedrine } \\
731.4 \pm 2.3\end{array}$} & \multirow{2}{*}{$\begin{array}{l}\begin{array}{l}\text { Pseudoephe } \\
\text { drine }\end{array} \\
242.8 \pm 2.3\end{array}$} \\
\hline $\mathbf{A}^{*}$ & Batch No.I & & & & & & & \\
\hline & Batch No.2 & $440.8 \pm 1.5$ & $342.1 \pm 2.7$ & $89.7 \pm 0.5$ & $59.8 \pm 0.5$ & $542.6 \pm 2.6$ & $938.5 \pm 8.8$ & $322.4 \pm 3.1$ \\
\hline & Batch No.3 & $738.7 \pm 4.1$ & $353.8 \pm 3.1$ & $100.2 \pm 0.7$ & $131.4 \pm 0.9$ & $1558.3 \pm 12.6$ & $1162.0 \pm 8.1$ & $318.6 \pm 2.0$ \\
\hline & Mean $( \pm S D)$ & $517.9 \pm 194.1$ & $322.1 \pm 45.1$ & $86.8 \pm 15.1$ & $81.1 \pm 43.7$ & $837.4 \pm 627.7$ & $944.0 \pm 215.4$ & $294.6 \pm 44.9$ \\
\hline \multirow[t]{4}{*}{ B* } & Batch No.I & $733.4 \pm 5.8$ & $423.1 \pm 2.0$ & $72.1 \pm 3.8$ & $63.2 \pm 1.0$ & $1584.3 \pm 12.2$ & $2121.0 \pm 7.4$ & $482.6 \pm 4.2$ \\
\hline & Batch No.2 & $2273.4 \pm 21.4$ & $1339.6 \pm 4.2$ & $183.4 \pm 1.7$ & $302.6 \pm 0.7$ & $3314.6 \pm 14.6$ & $2863.4 \pm 21.8$ & $891.4 \pm 3.7$ \\
\hline & Batch No.3 & $1708.6 \pm 11.6$ & $1030.7 \pm 6.5$ & $149.7 \pm 0.9$ & $228.7 \pm 0.8$ & $3276.5 \pm 23.9$ & $1822.6 \pm 0.7$ & $609.7 \pm 3.1$ \\
\hline & Mean $( \pm S D)$ & $\begin{array}{l}1571.8 \pm \\
779.1\end{array}$ & $931.1 \pm 466.3$ & $135.1 \pm 57.1$ & $198.2 \pm 122.6$ & $\begin{array}{l}2725.1 \pm \\
988.2\end{array}$ & $\begin{array}{l}2269.0 \pm \\
536.0\end{array}$ & $661.2 \pm 209.2$ \\
\hline \multirow[t]{3}{*}{ C* } & Batch No.I & $1202.8 \pm 14.0$ & $4587.7 \pm 16.1$ & $224.1 \pm 0.57$ & $138.3 \pm 0.8$ & $5917.4 \pm 39.1$ & $2886.8 \pm 0.7$ & $743.7 \pm 0.8$ \\
\hline & Batch No.2 & $1479.4 \pm 11.5$ & $1672.4 \pm 10.4$ & $149.6 \pm 0.7$ & $162.6 \pm 0.1$ & $18 \mid 4.2 \pm 5.4$ & $2293.7 \pm 0.2$ & $639.6 \pm 4.5$ \\
\hline & Mean $( \pm S D)$ & $1341.1 \pm 19.6$ & $\begin{array}{l}3130.1 \pm \\
2061.4\end{array}$ & $186.9 \pm 52.7$ & $151.5 \pm 18.6$ & $\begin{array}{l}3865.8 \pm \\
2901.4\end{array}$ & $\begin{array}{l}2590.3 \pm \\
419.4\end{array}$ & $691.7 \pm 10.7$ \\
\hline \multirow[t]{8}{*}{ D** } & Batch No.I & $5913.6 \pm 48.5$ & $4899.4 \pm 25.5$ & $323.6 \pm 1.9$ & $324.1 \pm 0.6$ & $5087.1 \pm 45.3$ & $3740.4 \pm 1.0$ & $902.4 \pm 8.1$ \\
\hline & Batch No.2 & $3417.7 \pm 18.5$ & $434 I .6 \pm 24.7$ & $238.9 \pm 0.6$ & $179.6 \pm 0.3$ & $4958.3 \pm 15.4$ & $3172.6 \pm 0.4$ & $1002.4 \pm 6.1$ \\
\hline & Batch No.3 & $3711.5 \pm 18.6$ & $4 \mid 24.8 \pm 32.5$ & $271.4 \pm 2.6$ & $242.4 \pm 0.9$ & $4079.6 \pm 11.8$ & $4577.8 \pm 0.8$ & $1203.8 \pm 6.3$ \\
\hline & Batch No.4 & $3889.3 \pm 19.4$ & $3128.7 \pm 21.0$ & $287.6 \pm 2.0$ & $263.7 \pm 0.8$ & $4189.3 \pm 20.5$ & $4329.6 \pm 0.4$ & $969.7 \pm 5.2$ \\
\hline & Batch No.5 & $4193.2 \pm 17.2$ & $3623.3 \pm 26.1$ & $304.4 \pm 2.7$ & $269.9 \pm 0.7$ & $3958.7 \pm 17.0$ & $3820.3 \pm 0.4$ & $1134.3 \pm 8.5$ \\
\hline & Batch No.6 & $4362.7 \pm 18.6$ & $3411.7 \pm 14.0$ & $313.2 \pm 2.3$ & $324.1 \pm 0.5$ & $4672.7 \pm 20.1$ & $2617.8 \pm 0.8$ & $933.1 \pm 4.9$ \\
\hline & Batch No.7 & $4438.6 \pm 31.1$ & $3462.6 \pm 7.6$ & $327.8 \pm 2.3$ & $317.8 \pm 0.6$ & $4729.7 \pm 8.5$ & $2624.0 \pm 0.8$ & $959.7 \pm 8.0$ \\
\hline & Mean $( \pm S D)$ & $\begin{array}{l}4275.2 \pm \\
809.1\end{array}$ & $\begin{array}{l}3856.0 \pm \\
623.4\end{array}$ & $295.3 \pm 31.8$ & $274.5 \pm 53.2$ & $\begin{array}{l}4525.0 \pm \\
447.1\end{array}$ & $\begin{array}{l}35554.6 \pm \\
779.1\end{array}$ & $\begin{array}{l}1015.1 \pm \\
111.5\end{array}$ \\
\hline
\end{tabular}

Values are means \pm SD of triplicate tests. Single factor ANOVA was performed on each component. Contents of each compound showed significant differences among the four manufacturers $(P<0.05)$. * Significant differences were observed in different sample batches $(P<0.05)$. ** No significant difference was observed in the contents of each component in seven batches of GT from manufacturer D (P > 0.05).

The quality of commercially available concentrated GT granules from the four manufacturers was evaluated by the established method. Typical chromatograms are shown in Figure 2. Although different samples from the four manufacturers showed a similar chromatographic profile, the peak areas were significantly varied. The contents of seven markers in different samples from different manufacturers were all within the ranges of the linear regression and much higher than LOQs (Table 4). In all samples, the major components were glycyrrhizin, puerarin, paeoniflorin and ephedrine. However, the contents of the measured constituents varied among batches within the GT granule from the same manufacturer. For example, the RSD for the samples from manufacturer D was less than $20 \%$, which is an acceptable variation for a botanical product, whereas the RSDs for the samples from manufacturer A for the seven markers were 14.0-74.9\% (Table 4). The average content of puerarin and glycyrrhizin in samples from manufacturer D, which showed the highest amount of markers, were six-fold higher than those from manufacturer $\mathrm{A}$. In addition, daily intake amounts according to the daily recommended dosage (Table 1) were compared (Figure 3 ). The total daily intake amounts of the seven marker components in the samples from manufacturers A, B, C and D were 38.2, 83.2, 137.9 and $173.8 \mathrm{mg}$ respectively.

\section{Discussion}

Previously, two HPLC systems were applied for the determination of components in the decoction of GT using a $\mathrm{C}_{18}$ column with an ion-pair mobile phase to analyze ephedrine alkaloids [14], and a gradient mobile phase to separate non-alkaloids components [15]. These methods are time consuming and not suitable for GT granules. A MEKC method has been used for the determination of seven components in GT granules. Although the analysis time for the MEKC method was short, the reported recovery, precision and sensitivity were low. In our study, different types of columns, such as $\mathrm{C}_{18}$ and $\mathrm{C}_{8}$, were compared. The phenyl column showed a better simultaneous separation of all seven chemical markers.

Apart from puerarin and daidzein, the chemical structures of the markers are different (Figure 1). As the maximum absorbance wavelengths of these components are different, it is difficult to obtain a sensitive determination for all components with a single wavelength. Therefore, multiwavelength detection was selected in order to obtain a sensitive determination for all components. The validated results showed that the proposed HPLC method was sensitive, precise, accurate and reliable for the quality assessment of GT granules.

The herbal compositions of the GT granules from the four manufacturers are similar (Table 1); however, the statisti- 


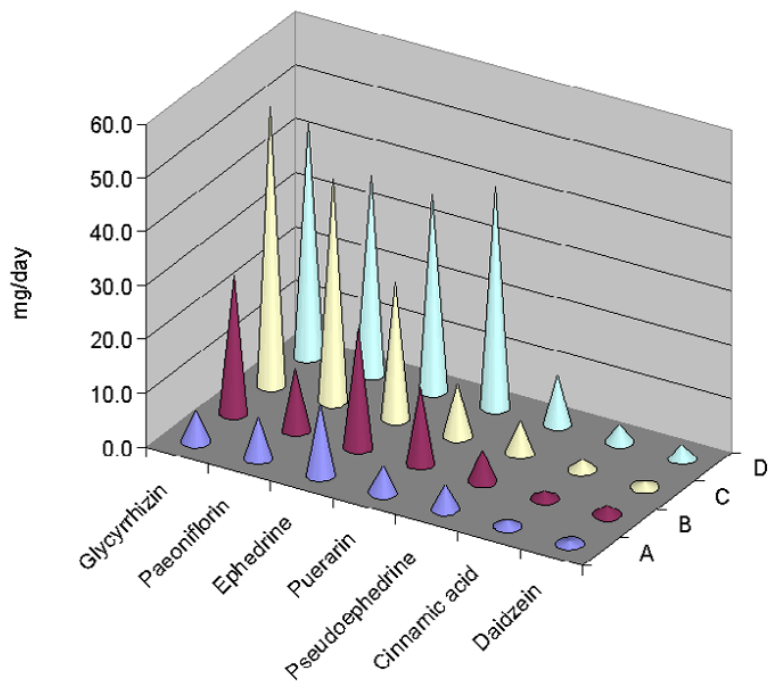

Figure 3

Comparison of daily intake amounts of the seven compounds in GT granules from four manufacturers. Letters $A, B, C$ and $D$ denote the four manufacturers. Values are means \pm SD of batches from each manufacturer. Single factor ANOVA was performed on each compound. Contents of each compound showed significant differences among the four manufacturers $(P<0.05)$.

cal analysis indicated that the contents of seven marker compounds (Table 4) and daily intake amounts of the marker compounds (Figure 3) were significantly different among the four manufacturers $(\mathrm{P}<0.05)$. Moreover, significant differences were observed in different sample batches from manufacturers $\mathrm{A}, \mathrm{B}$ and $\mathrm{C}(\mathrm{P}<0.05)$ respectively. Full assessment of the quality of GT granule preparations is therefore warranted. On the other hand, no significant differences $(\mathrm{P}>0.05)$ of the contents of the marker compounds were found in samples of different batches from manufacturer $\mathrm{D}$, an indication of better quality consistency from this manufacturer.

\section{Conclusion}

A multi-component HPLC method with a simple extraction procedure has been developed for the simultaneous quantitation of seven markers in commercially available concentrated GT granules. Results indicate that the present method was precise, accurate and reliable in controlling the quality of GT granule. The quantitative results show that the contents of the seven markers in GT granules were significantly varied among the four manufacturers, as well as among batches from the same manufacturer. We conclude that this HPLC method is a reliable and simple method for the quality evaluation of concentrated GT granules.

\section{Competing interests}

The author(s) declare that they have no competing interests.

\section{Authors' contributions}

JS and YY performed sample collection, method development, validation, sample analysis and manuscript drafting. CQ and QH participated in the design of the study, chemical markers collection and manuscript drafting. HX conceived the idea of the study and participated in its design and coordination. All authors approved the final manuscript.

\section{Acknowledgements}

This research has been funded by the Hong Kong Jockey Club Charities Trust.

\section{References}

I. Bensky D, Barolet R: Formulas that release the exterior. In Chinese Herbal Medicine Formulas \& Strategies Eastland Press; 1990:5I-2.

2. Chueh FS, Chang CP, Chio CC, Lin MT: Puerarin acts through brain serotonergic mechanisms to induce thermal effects. J Pharmacol Sci 2004, 96:420-7.

3. Muraoka K, Yoshida S, Hasegawa K, Nakanishi N, Fukuzawa I, Tomita A, Cyong JC: A pharmacologic study on the mechanism of action of Kakkon-to: body temperature elevation and phagocytic activation of macrophages in dogs. J Altern Complement Med 2004, 1 0:84I-9.

4. Nagasaka K, Kurokawa M, Imakita M, Terasawa K, Shiraki K: Efficacy of Kakkon-to, a traditional herb medicine, in herpes simplex virus type I infection in mice. J Med Virol 1995, 46:28-34.

5. Ikeda N, Hayasaka S, Nagaki Y, Hayasaka Y, Kadoi C, Matsumoto M Effects of traditional Sino-Japanese herbal medicines on aqueous flare elevation after small-incision cataract surgery. J Ocul Pharmacol Ther 200I, I 7:59-65.

6. Sugiyama A, Takahara A, Satoh Y, Yoneyama M, Saegusa Y, Hashimoto K: Cardiac effects of clinically available Kampo medicine assessed with canine isolated, blood-perfused heart preparations. Jpn J Pharmacol 2002, 88:307-I3.

7. Ozaki Y: Studies on antiinflammatory effect of Japanese Oriental medicines (Kampo medicines) used to treat inflammatory diseases. Biol Pharm Bull 1995, I 8:559-62.

8. Ikeda N, Hayasaka S, Nagaki Y, Hayasaka Y, Kadoi C, Matsumoto M: Effects of Kakkon-to and Sairei-to on aqueous flare elevation after complicated cataract surgery. Am J Chin Med 2002, 30:347-53.

9. Kurokawa M, Tsurita M, Brown J, Fukuda Y, Shiraki K: Effect of interleukin- 12 level augmented by Kakkon-to, a herbal medicine, on the early stage of influenza infection in mice. Antiviral Res 2002, 56:183-8.

10. Lu XR, Gao E, Xu LZ, Li HZ, Kang B, Chen WN, Chen SM, Chai XS: Puerarin beta-adrenergic receptor blocking effect. Chin Med $J$ (Engl) 1987, I00:25-9.

II. Yan LP, Chan SW, Chan AS, Chen SL, Ma XJ, Xu HX: Puerarin decreases serum total cholesterol and enhances thoracic aorta endothelial nitric oxide synthase expression in dietinduced hypercholesterolemic rats. Life Sci 2006, 79:324-30.

12. Woodman OL, Boujaoude M: Chronic treatment of male rats with daidzein and 17 beta-oestradiol induces the contribution of EDHF to endothelium-dependent relaxation. $\mathrm{Br} J$ Pharmacol 2004, | 4 I:322-8.

13. Lian WY, Feng RZ, Chen BZ, Zhou YP, Su XL, Zhong Y, Gu ZP, Xu Q, Fu GX: Studies on Radix Puerariae. In Species Systematization and Quality Evaluation of Commonly Used Chinese Traditional Drugs, North-China Etidion Volume I. Edited by: Lou ZC. Qin B: Peking Univ Med Press; 1995:406-16.

14. Okamura N, Miki H, Harada T, Yamashita S, Masaoka Y, Nakamoto Y, Tsuguma M, Yoshitomi H, Yagi A: Simultaneous determination of ephedrine, pseudophedrine, norephedrine and methyl- 
ephedrine in Kampo medicines by high-performance liquid chromatography. J Pharm Biomed Anal 1999, 20:363-72.

15. Okamura N, Miki H, Orii H, Masaoka Y, Yamashita S, Kobayashi H, Yagi A: Simultaneous high-performance liquid chromatographic determination of puerarin, daidzein, paeoniflorin, liquiritin, cinnamic acid, cinnamaldehyde and glycyrrhizin in Kampo medicines. J Pharm Biomed Anal 1999, 19:603-12.

16. Huang HY, Hsieh YZ: Determination of puerarin, daidzein, paeoniflorin, cinnamic acid, glycyrrhizin, ephedrine, and [6]-gingerol in Gegen Tang by micellar electrokinetic chromatography. Anal Chimi Acta 1997, 35 I:49-55.

Publish with Biomed Central and every scientist can read your work free of charge

"BioMed Central will be the most significant development for disseminating the results of biomedical research in our lifetime. " Sir Paul Nurse, Cancer Research UK

Your research papers will be:

- available free of charge to the entire biomedical community

- peer reviewed and published immediately upon acceptance

- cited in PubMed and archived on PubMed Central

- yours - you keep the copyright 